\title{
Diversely Substituted Indoloazepinones and Indoloazocinones: A Post-Ugi Gold-Catalyzed Regioselective Carbocyclization Approach
}

\author{
Dipak D. Vachhani ${ }^{a}$ \\ Amit Kumara,b \\ Sachin G. Modha*a,1 \\ Sunil K. Sharmab \\ Virinder S. Parmar ${ }^{b}$ \\ Erik V. Van der Eycken*a \\ a Laboratory for Organic \& Microwave-Assisted Chemistry \\ (LOMAC), Department of Chemistry, University of Leuven \\ (KU Leuven), Celestijnenlaan 200F, 3001 Leuven, Belgium \\ b Bioorganic Laboratory, Department of Chemistry, University \\ of Delhi, Delhi 110 007, India \\ erik.vandereycken@chem.kuleuven.be
}

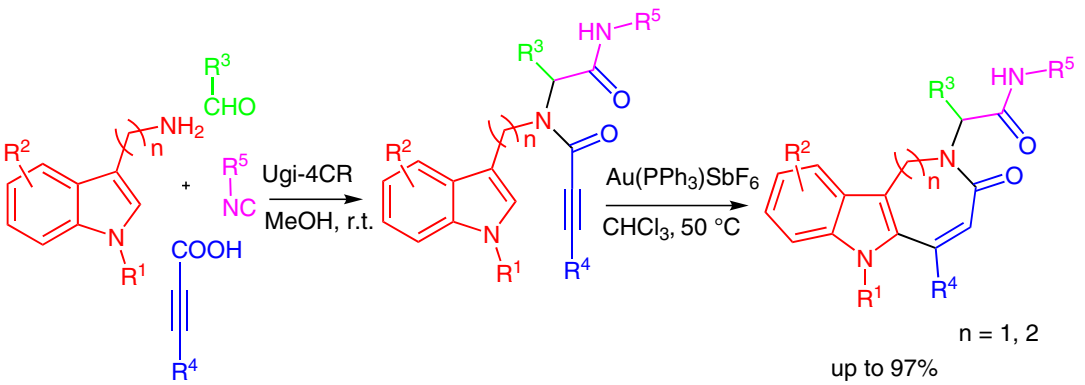

Received: 27.10.2014

Accepted after revision: 15.01.2015

Published online: 26.02 .2015

DOI: 10.1055/s-0034-1379894; Art ID: ss-2014-z0651-op

Abstract A post-Ugi gold-catalyzed regioselective intramolecular carbocyclization strategy is reported. A better cationic gold system, compared to our previous report, has been optimized and utilized. The method presented exhibits excellent functional group compatibility including bulky substitutions on alkynes and allows the direct access to indoloazepinones and indoloazocinones in good to excellent yields.

Key words post-Ugi, gold, indoloazepinone, indoloazocinone, carbocyclization

Synthetic molecules based on natural products play a major role in the discovery of new therapeutic agents for treating diseases. ${ }^{2}$ In this context, the indoloazepinones skeleton is the core of important natural products such as the indole alkaloids tronocarpine $(\mathbf{I}),{ }^{3}$ the paullones $\mathbf{I I},{ }^{4}$ and the malassezindoles III $^{5}$ (Figure 1 ). Furthermore, some azepinoindoles possess anticancer activity ${ }^{4 \mathrm{~b}, 6}$ and are found to be active against central nervous system diseases. ${ }^{7}$ Owing to their medicinal value, the development of novel and efficient methods for constructing this heteroarene ring has received continuous interest in recent years., ${ }^{8,9}$ On the other hand, gold-catalyzed hetereoannulations and carbocyclizations are of contemporary importance due to the selective and efficient activation of alkynes towards a wide range of nucleophiles. ${ }^{10}$

Recently, we reported a concise route to azocino[5,4$b$ ]indol-4-one via a sequential Ugi gold-catalyzed intramolecular hydroarylation. ${ }^{11}$ In continuation of this work and as a result of our interest in transition-metal catalysis ${ }^{12}$ and multicomponent reactions ${ }^{13}$ for the synthesis of diversely substituted heterocycles, we elaborated a post-Ugi goldcatalyzed regioselective carbocyclization approach for the synthesis of indoloazepinones. Moreover, this protocol al-

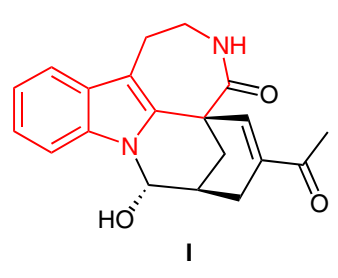<smiles>[R]c1ccc2[nH]c3c(c2c1)CC(=O)Nc1ccccc1-3</smiles>

Ila $\mathrm{R}=\mathrm{H}$

Illb $\mathrm{R}=\mathrm{Br}$

Ilc $\mathrm{R}=\mathrm{NO}_{2}$<smiles>[R][C@]1(c2c[nH]c3ccccc23)C(=O)N[C@@H](C(=O)O)Cc2c1[nH]c1ccccc21</smiles>

IIla $\mathrm{R}=\mathrm{OH}$ IIIb $R=H$<smiles>[R]C1=CC(=O)N([R7])Cc2c1n(C)c1ccccc21</smiles>

IV
Figure 1 Tronocarpine (I), paullones II, malassezindoles III, this work IV

lows the synthesis of indoloazocinones bearing sterically hindered substituents on the alkyne, which was not possible to achieve using our previously reported protocol. ${ }^{11}$

Ugi four-component reaction (Ugi-4CR) ${ }^{14}$ of indolemethylamine 1a, benzaldehyde (2a), but-2-ynoic acid (3a), and 1,1,3,3-tetramethylbutyl isocyanide (4a) in methanol at room temperature furnishes the corresponding Ugi adduct $\mathbf{5 a}$ in $95 \%$ yield. This was further used for investigating the intramolecular carbocyclization (Table 1).

The application of $5 \mathrm{~mol} \%$ of $\mathrm{Au}\left(\mathrm{PPh}_{3}\right) \mathrm{OTf}$ in $\mathrm{CDCl}_{3}$ at room temperature for 24 hours, resulted in $61 \%$ of indoloazepinone 6a, as measured by ${ }^{1} \mathrm{H}$ NMR spectroscopy (Table 1 , entry 1). No amelioration was observed using $\mathrm{Au}\left(\mathrm{PPh}_{3}\right) \mathrm{BF}_{4}$, $\mathrm{AuSbF}_{6}$, and $\mathrm{Au}(\mathrm{JohnPhos}) \mathrm{SbF}_{6}$ as catalyst while the use of $\mathrm{Yb}(\mathrm{OTf})_{3}$ resulted in only traces of the product (entries 2- 
Table 1 Optimization of the Intramolecular Carbocylization ${ }^{\mathrm{a}}$

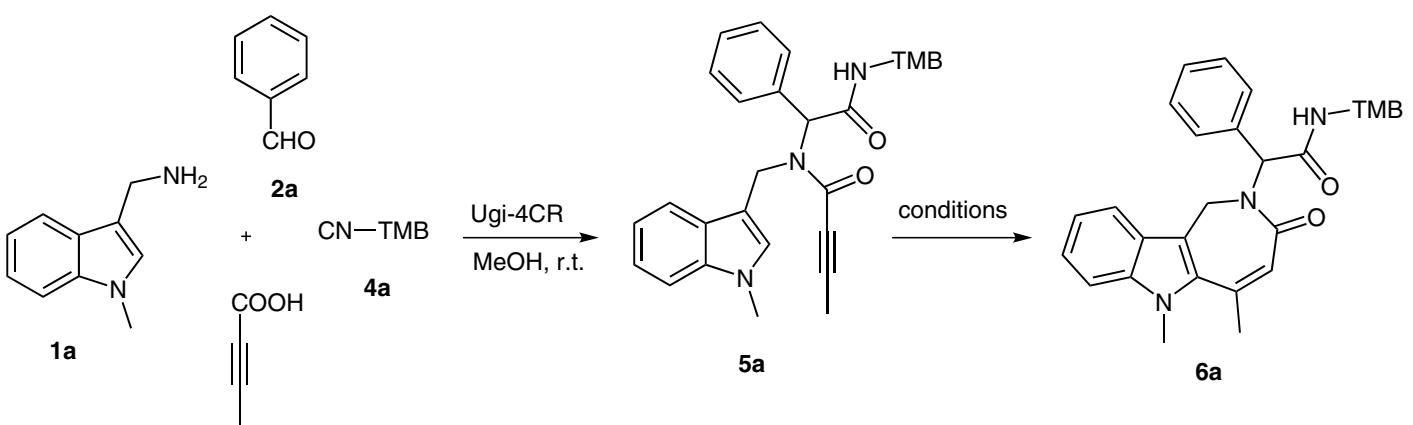

3a

\begin{tabular}{|c|c|c|c|c|c|}
\hline Entry & Catalyst & Solvent & $\operatorname{Temp}\left({ }^{\circ} \mathrm{C}\right)$ & Time (h) & Yield (\%) \\
\hline 1 & $\mathrm{Au}\left(\mathrm{PPh}_{3}\right) \mathrm{OTf}$ & $\mathrm{CDCl}_{3}$ & r.t. & 24 & 61 \\
\hline 2 & $\mathrm{Au}\left(\mathrm{PPh}_{3}\right) \mathrm{BF}_{4}$ & $\mathrm{CDCl}_{3}$ & r.t. & 24 & 38 \\
\hline 3 & $\mathrm{AuSbF}_{6}$ & $\mathrm{CDCl}_{3}$ & r.t. & 24 & 23 \\
\hline 4 & $\mathrm{Yb}(\mathrm{OTf})_{3}$ & $\mathrm{CDCl}_{3}$ & r.t. & 24 & traces \\
\hline 5 & $\mathrm{Au}(\mathrm{L}) \mathrm{SbF}_{6}{ }^{\mathrm{c}}$ & $\mathrm{CDCl}_{3}$ & r.t. & 24 & 81 \\
\hline 6 & $\mathrm{Au}\left(\mathrm{PPh}_{3}\right) \mathrm{SbF}_{6}$ & $\mathrm{CDCl}_{3}$ & r.t. & 24 & 86 \\
\hline 7 & $\mathrm{Au}\left(\mathrm{PPh}_{3}\right) \mathrm{SbF}_{6}$ & $\mathrm{CD}_{3} \mathrm{CN}$ & r.t. & 24 & 59 \\
\hline 8 & $\mathrm{Au}\left(\mathrm{PPh}_{3}\right) \mathrm{SbF}_{6}$ & THF- $d_{8}$ & r.t. & 24 & 48 \\
\hline 9 & $\mathrm{Au}\left(\mathrm{PPh}_{3}\right) \mathrm{SbF}_{6}$ & $\mathrm{CDCl}_{3}$ & 50 & 4 & $90(88)^{c}$ \\
\hline 10 & $\mathrm{Au}\left(\mathrm{PPh}_{3}\right) \mathrm{SbF}_{6}$ & $\mathrm{CDCl}_{3}$ & 50 & 24 & $84^{e}$ \\
\hline
\end{tabular}

${ }^{a}$ All reactions were run on a $0.1 \mathrm{mmol}$ scale of 5 a using $5 \mathrm{~mol} \%$ of catalyst; TMB $=1,1,3,3$-tetramethylbutyl.

${ }^{b}$ Yields were determined on the basis of ${ }^{1} \mathrm{H}$ NMR analysis using 3,4,5-trimethoxybenzaldehyde as an internal standard.

${ }^{c} \mathrm{~L}=2$-(Di-tert-butylphosphino)biphenyl (JohnPhos).

d Isolated yield.

e Amount of catalyst used: 2 mol\%.

5). Interestingly, the application of $\mathrm{Au}\left(\mathrm{PPh}_{3}\right) \mathrm{SbF}_{6}$ substantially enhanced the yield of $\mathbf{6 a}$ to $86 \%$ (entry 6 ). Among the solvents screened, $\mathrm{CDCl}_{3}$ was found to be the solvent of choice (entries 7 and 8). Reaction with $\mathrm{Au}\left(\mathrm{PPh}_{3}\right) \mathrm{SbF}_{6}$ at $50{ }^{\circ} \mathrm{C}$ sped up the reaction and increased the yield to $90 \%$ (entry 9). A decrease of the catalyst loading to $2 \mathrm{~mol} \%$ resulted in a lower yield even after a prolonged reaction time (entry 10).

Having optimized the conditions for the intramolecular carbocyclization (Table 1, entry 9), the scope and limitations of the protocol were explored. A diversely substituted set of Ugi adducts was synthesized and subjected to the reaction conditions.

The endo-dig cyclization proceeds smoothly giving the indoloazepinones in good to excellent yields (Table 2). The reaction is feasible with Ugi adducts derived from aliphatic as well as aromatic aldehydes (Table 2, entries 1-6). Interestingly, bulky substituents on the alkyne like ethyl, isopropyl, and aryl are well tolerated (entries 7-10). However, the Ugi adduct 5k containing a tert-butyl substituent, failed to give the desired cyclized product (entry 11). As expected, the terminal alkyne $\mathbf{5 1}$ gave the 6 -exo-dig product $\mathbf{6 1}$, due to the involvement of a gold carbene intermediate (entry 12). ${ }^{11,15}$

Inspired by the observation that Ugi adduct with bulky substituent like phenyl can also undergo endo-dig carbocyclization at high temperature, we were keen to investigate this modified protocol on previously reported but unsuccessful intramolecular hydroarylation for the synthesis of indoloazocinones. ${ }^{11,16}$ To our great satisfaction, the method works efficiently leading to indoloazocinones in good yields (Table 3). A comparative study of the new reaction conditions with the previously reported $\mathrm{Au}\left(\mathrm{PPh}_{3}\right) \mathrm{OTf}$-catalyzed hydroarylation was performed. In most cases, the current protocol proved to be superior delivering the desired indoloazocinones with improved yields. ${ }^{11}$ Nevertheless, the tertbutyl appears to be too bulky to give the cyclized product (Table 3, entry 7).

Based on our observations and literature reports ${ }^{15,17} \mathrm{a}$ plausible mechanism is depicted in Scheme 2 . The counterion $\mathrm{SbF}_{6}{ }^{-}$is believed to be completely dissociated from gold in solution, thus making it more cationic as compared to OTf-. Coordination of this cationic gold with the alkyne in $\mathbf{5}$ 
generates intermediate $\mathbf{A}$. In the case of an internal alkyne, the nucleophilic attack of the 3-position of the indole on the activated alkyne occurs in an endo-dig fashion generating intermediate $\mathbf{B}$. This is followed by a 1,2-shift to furnish intermediate $\mathbf{C}$, which upon deprotonation and protodeauration results in the formation of indoloazepinone $\mathbf{6}$. In the case of a terminal alkyne the nucleophilic attack of the 3position of the indole occurs in an exo-dig fashion generating intermediate $\mathbf{B}^{\prime}$. After 1,2-shift, deprotonation and protodeauration, indolopyridinone $\mathbf{6 i}$ is formed.
In summary, we have elaborated an improved cationic gold-catalyzed post-Ugi intramolecular carbocyclization protocol for the synthesis of indoloazepinones and indoloazocinones. Contrary to our previous work, the new methodology works equally well with bulky substituents delivering the compounds in good yields. However, conditions are still not able to tolerate the tert-butyl group, and attempts to further improve the protocol are in progress.

Table 2 Scope and Limitations of the Intramolecular Carbocyclization ${ }^{\mathrm{a}}$

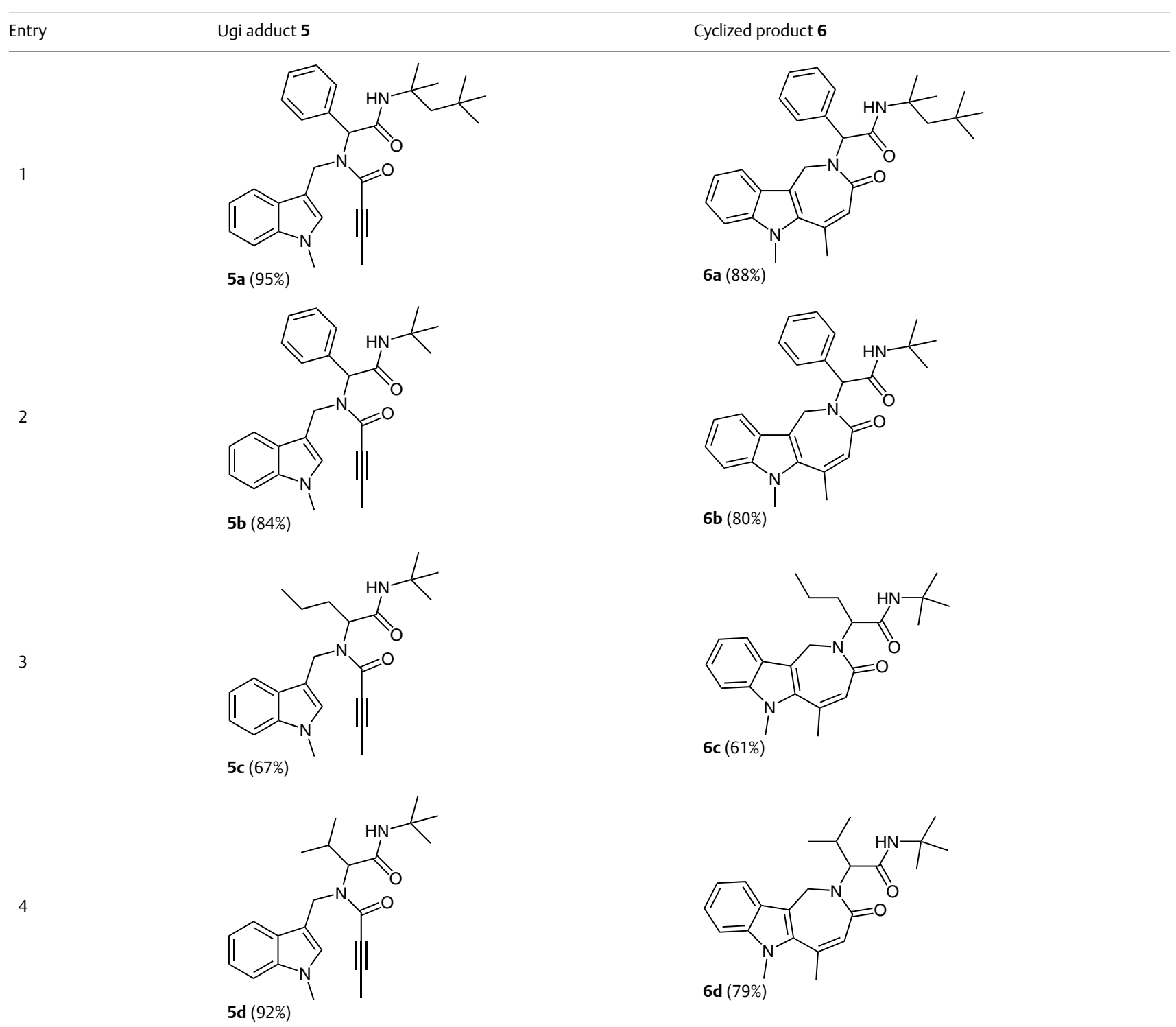


Synthesis

D. D. Vachhani et al.

Paper

Table 2 (continued)

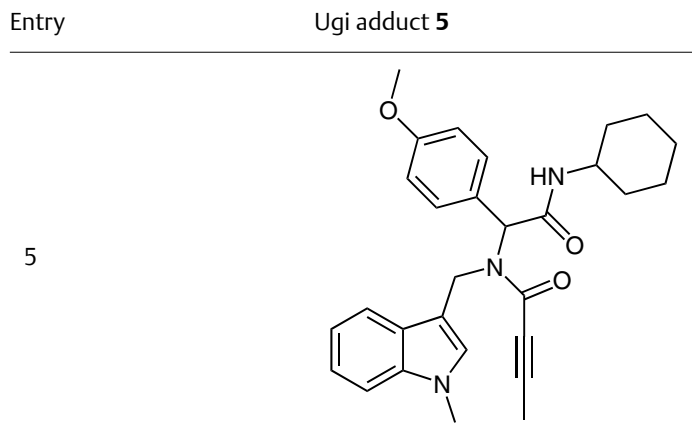

5 e $(47 \%)$

6

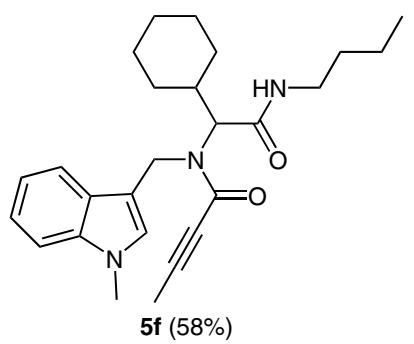

5f (58\%)

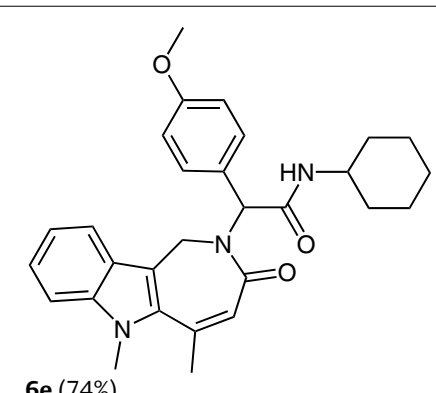

6e $(74 \%)$

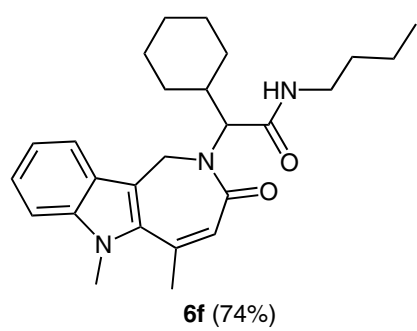

$6 f(74 \%)$
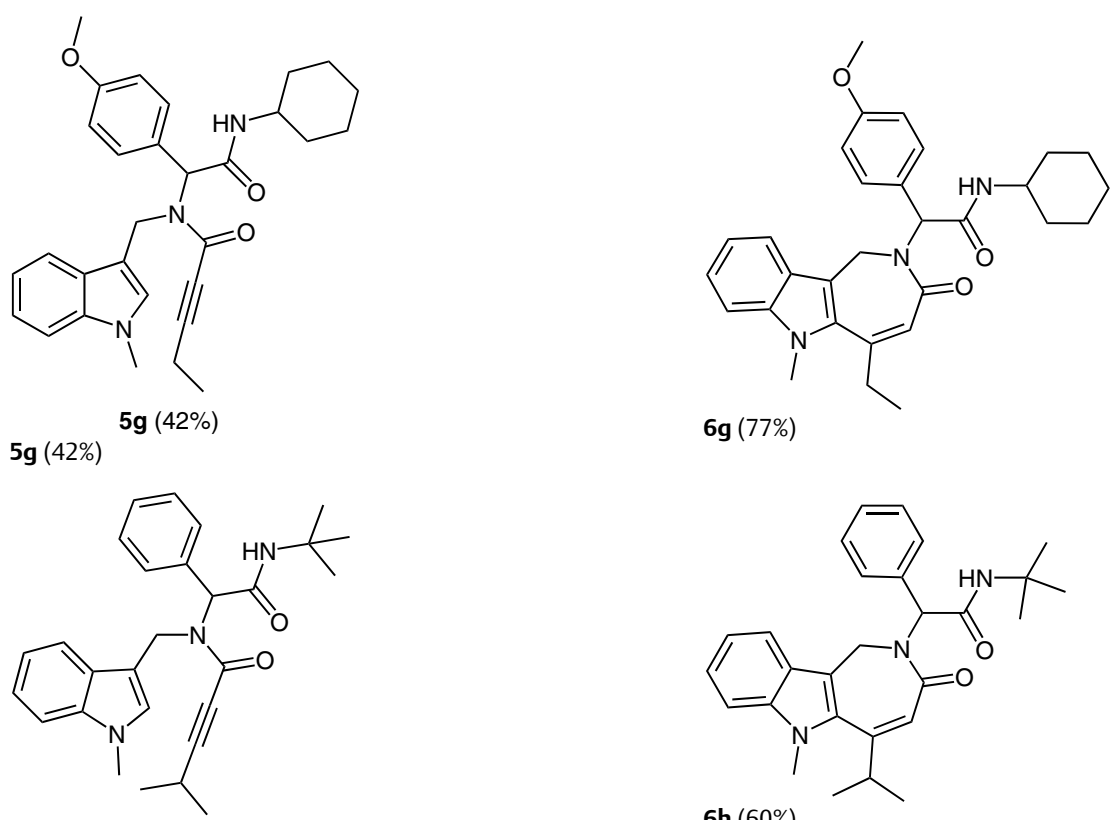

$\mathbf{6 g}(77 \%)$

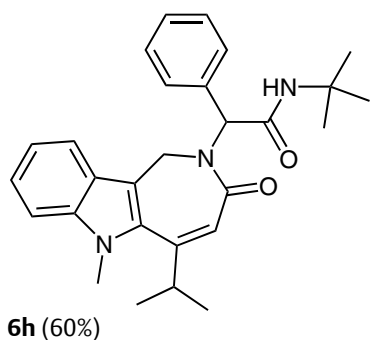

$5 h(83 \%)$

6h (60\%)
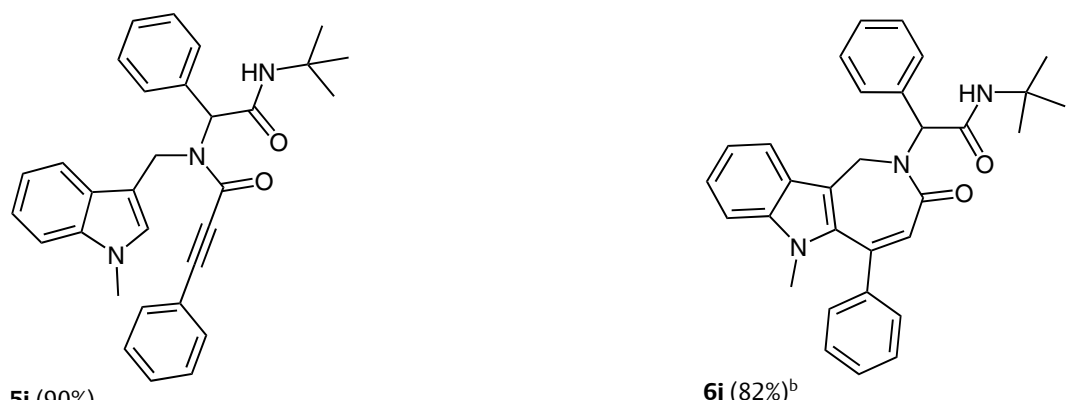

$5 \mathbf{i}(90 \%)$

6i $(82 \%)^{b}$ 
Table 2 (continued)

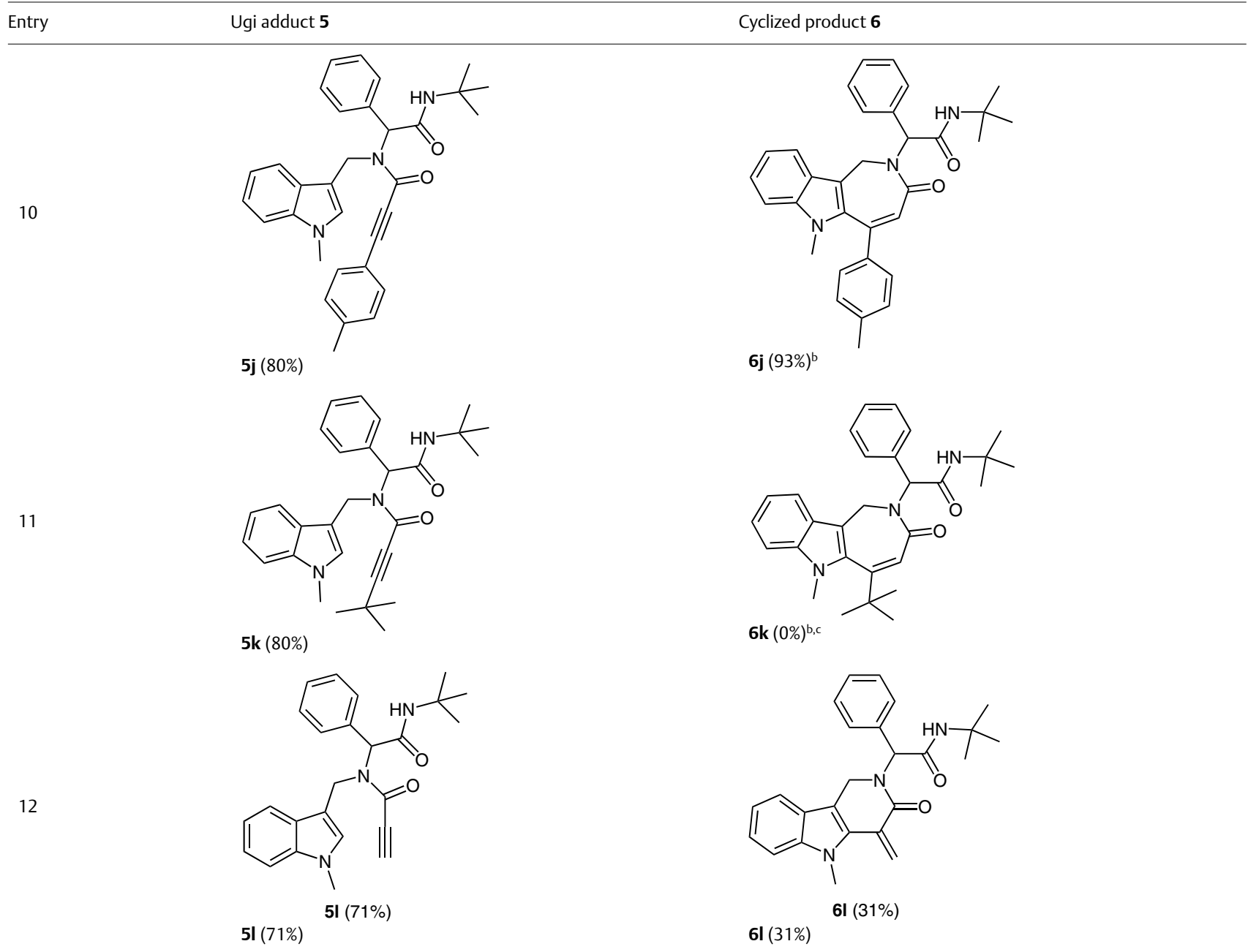

${ }^{a}$ Conditions for the Ugi-4CR: indolemethylamine $\mathbf{1}$ (1 mmol), aldehyde $\mathbf{2}$ (1.2 equiv), alkynoic acid $\mathbf{3}$ (1.2 equiv), and isonitrile 4 (1.2 equiv) in MeOH at r.t. for 24 h; conditions for the intramolecular carbocyclization: Ugi adduct $5(0.2 \mathrm{mmol}), \mathrm{Au}\left(\mathrm{PPh}_{3}\right) \mathrm{Cl}(5 \mathrm{~mol} \%)$, and $\mathrm{AgSbF}_{6}(5 \mathrm{~mol} \%)$ in $\mathrm{CHCl} 3(2 \mathrm{~mL})$ at $50{ }^{\circ} \mathrm{C} \mathrm{for} 3-6 \mathrm{~h}$.

b The reaction was performed at $80^{\circ} \mathrm{C}$.

c An unidentified mixture of products were obtained.

All the starting materials, reagents, and catalysts were purchased from Aldrich or Acros and used as such. For TLC, analytical TLC plates (Alugram SIL G/UV254 and 70-230 mesh silica gel (E. Merck) were used. Column chromatography was performed using silica gel (Merck, 60-120 mesh size). Anhydrous solvents were purchased from Acros Organics and stored over molecular sieves. The chromatographic solvents used for isolation/purification of compounds were distilled prior to use. The chromatographic solvents are mentioned as volume:volume ratios. Reactions were typically run in oven-dried screw-cap vial under an inert atmosphere.

${ }^{1} \mathrm{H}(300 \mathrm{MHz})$ and ${ }^{13} \mathrm{C}(75.5 \mathrm{MHz})$ NMR spectra were recorded on a $400 \mathrm{MHz}$ and $300 \mathrm{MHz}$ instruments using $\mathrm{CDCl}_{3}$ and DMSO- $d_{6}$ as a solvent. The ${ }^{1} \mathrm{H}$ and ${ }^{13} \mathrm{C}$ chemical shifts are reported in parts per million relative to TMS using the residual solvent signal as the internal reference. Standard abbreviations were used to designate chemical shift multiplicities. ${ }^{13} \mathrm{C}$ NMR spectra are proton decoupled. Melting points were determined on a digital Barnsted Electrothermal 9200 apparatus and are uncorrected. Mass spectra were recorded by using a Kratos MS50TC and a Kratos Mach III system. The ion source temperature was $150-250{ }^{\circ} \mathrm{C}$, as required. High-resolution EI-mass spectra were performed with a resolution of 10000 . The low-resolution spectra were obtained with a HP5989A MS instrument. The low-resolution ESI-MS were obtained with a Thermo Scientific instrument.

\section{Ugi Products 5a-s and 8a-g; General Procedure}

To a solution of substituted amine $\mathbf{1}$ or $\mathbf{7 a}, \mathbf{b}$ ( $1 \mathrm{mmol}, 1$ equiv) in $\mathrm{MeOH}(5 \mathrm{~mL})$ were added successively $\mathrm{Na}_{2} \mathrm{SO}_{4}(0.3 \mathrm{~g})$, aldehyde $\mathbf{2 a}-\mathbf{g}$ (1.2 equiv), alkynoic acid $\mathbf{3 a}-\mathbf{h}$ (1.2 equiv), and isonitrile $\mathbf{4 a - d}$ (1.2 equiv) in a $25 \mathrm{~mL}$ round-bottomed flask equipped with a magnetic stir bar. The reaction mixture was stirred at r.t. for $24 \mathrm{~h}$. After completion of the reaction, the mixture was diluted with EtOAc $(100 \mathrm{~mL})$ and the organic layer was washed with $\mathrm{H}_{2} \mathrm{O}(50 \mathrm{~mL})$ and brine $(50 \mathrm{~mL})$, dried $\left(\mathrm{MgSO}_{4}\right)$, and evaporated under reduced pressure. The residue was subjected to silica gel column chromatography (30-50\% EtOAc in heptane) to afford the respective desired products $\mathbf{5 a - 1}$ or $\mathbf{8 a - g}$ as solids. 
Table 3 Expanding the Scope of the Intramolecular Carbocyclization to Indoloazocinones and Comparison with the Previous Protocol

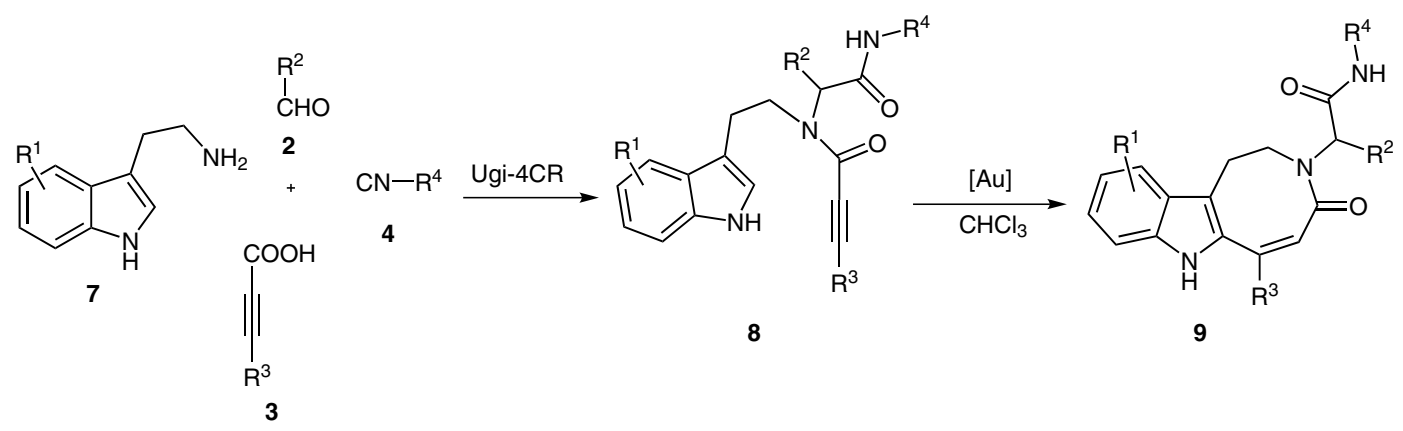

1<smiles>CC(C)(C)NC(=O)C(N(CCc1c[nH]c2ccccc12)C(=O)C#Cc1ccccc1)C(C)(C)C</smiles>

2<smiles>CC#CC(=O)N(CCc1c[nH]c2ccccc12)C(C(=O)NCCCC)c1c(Cl)cccc1Cl</smiles>

3<smiles>CC#CC(=O)N(CCc1c[nH]c2ccc(OC)cc12)C(C(=O)NC(C)(C)C)C1CCCCC1</smiles>

4<smiles>COc1ccc(C#CC(=O)N(CCc2c[nH]c3ccccc23)C(C(=O)NC2CCCCC2)c2ccccc2)cc1</smiles>

$8 a$<smiles>CC(C)(C)NC(=O)C(N1CCc2c([nH]c3ccccc23)/C(c2ccccc2)=C\C1=O)C(C)(C)C</smiles>

$8 b$ 91

$8 c$

8d<smiles>[Mg][Mg]</smiles><smiles>CCCCNC(=O)C(c1c(Cl)cccc1Cl)N1CCc2c([nH]c3ccccc23)/C(C)=C/C1=O</smiles><smiles>COc1ccc2[nH]c3c(c2c1)CCN(C(C(=O)NC(C)(C)C)C1CCCCC1)C(=O)/C=C\3C</smiles>

$9 a$

$80^{\mathrm{d}} \quad 0$

9b $\quad 94 \quad 80$

$9 c \quad 97 \quad 65$<smiles>COc1ccc(/C2=C/C(=O)N(C(C(=O)NC3CCCCC3)c3ccccc3)CCc3c2[nH]c2ccccc32)cc1</smiles>

9d
NA 
<smiles>CCCC(C(=O)NC(C)(C)C)N(CCc1c[nH]c2ccc(OC)cc12)C(=O)C#Cc1ccccc1</smiles>

$8 e$

76<smiles></smiles>

$9 e$ $84^{d}$

NA
6<smiles>CC(C)C#CC(=O)N(CCc1c[nH]c2ccccc12)C(C(=O)NC(C)(C)C)C1CCCCC1</smiles>

7<smiles>CC(C)(C)C#CC(=O)N(CCc1c[nH]c2ccccc12)C(C(=O)NC(C)(C)C)C1CCCCC1</smiles>

$8 f$

86<smiles>CC(C)/C1=C/C(=O)N(C(C(=O)NC(C)(C)C)C2CCCCC2)CCc2c1[nH]c1ccccc21</smiles>

$8 g$

84<smiles>CC(C)(C)NC(=O)C(C1CCCCC1)N1CCc2c([nH]c3ccccc23)C(C(C)(C)C)=CC1=O</smiles>

$N$-[(1-Methyl-1H-indol-3-yl)methyl]- $N$-[2-oxo-1-phenyl-2-(2,4,4trimethylpentan-2-yl-amino)ethyl]but-2-ynamide (5a)

Yield: $447 \mathrm{mg}$ (95\%); off-white solid; $\mathrm{mp} \mathrm{56-58}{ }^{\circ} \mathrm{C}$.

${ }^{1} \mathrm{H}$ NMR (300 MHz, $\mathrm{CDCl}_{3}$ ): $\delta=7.74-7.60(\mathrm{~m}, 1 \mathrm{H}), 7.48-7.33(\mathrm{~m}, 2.6$ H), 7.32-7.18 (m, 4.5 H), 7.17-7.03 (m, $1.5 \mathrm{H}), 6.91(\mathrm{~s}, 0.5 \mathrm{H}), 6.03(\mathrm{~s}$, $0.5 \mathrm{H}), 5.61(\mathrm{~s}, 0.5 \mathrm{H}), 5.28(\mathrm{~s}, 0.5 \mathrm{H}), 5.12(\mathrm{~d}, J=15.81 \mathrm{~Hz}, 0.5 \mathrm{H}), 4.85$ $(\mathrm{d}, J=15.84 \mathrm{~Hz}, 0.5 \mathrm{H}), 4.72(\mathrm{~d}, J=14.85 \mathrm{~Hz}, 0.5 \mathrm{H}), 4.27$ (d, $J=14.88$ $\mathrm{Hz}, 0.5 \mathrm{H}$ ), 3.78-3.64 (m, $3 \mathrm{H}), 2.04-1.91$ (m, $3 \mathrm{H}), 1.76-1.64$ (m, 0.5 H), $1.33(\mathrm{~d}, J=14.88 \mathrm{~Hz}, 0.5 \mathrm{H}), 1.24(\mathrm{~s}, 1.5 \mathrm{H}), 1.07(\mathrm{~s}, 1.5 \mathrm{H}), 0.97$ (s, $1.5 \mathrm{H}), 0.89-0.65(\mathrm{~m}, 10 \mathrm{H}), 0.50(\mathrm{~s}, 1.5 \mathrm{H})$.

${ }^{13} \mathrm{C} \mathrm{NMR}\left(75 \mathrm{MHz}, \mathrm{CDCl}_{3}\right): \delta=167.3,167.2,156.0,155.0,137.1,136.7$, 135.1, 134.7, 129.8, 129.7, 129.2, 128.8, 128.6, 128.3, 128.2, 127.9, 127.0, 126.8, 122.3, 122.0, 120.0, 119.6, 118.9, 110.4, 109.6, 109.5, 109.4, 91.9, 90.5, 74.0, 73.5, 67.0, 64.1, 55.0, 51.7, 50.4, 45.0, 37.5, $32.7,31.3,31.1$ (3), 28.4, 28.3, 28.0, 27.6, 4.1 (2).
HRMS: $m / z$ calcd for $\mathrm{C}_{30} \mathrm{H}_{37} \mathrm{~N}_{3} \mathrm{O}_{2}$ : 471.2886; found: 471.2876 .

2-(5,6-Dimethyl-3-oxoazepino[4,3-b]indol-2(1H,3H,6H)-yl)-2-phenyl- $N$-(2,4,4-trimethylpentan-2-yl)acetamide (6a); Typical Procedure

In a $10 \mathrm{~mL}$ screw cap vial charged with the Ugi adduct $\mathbf{5 a}(0.2 \mathrm{mmol}$, $94 \mathrm{mg}$ ) was added $\mathrm{AuPPh}_{3} \mathrm{Cl}$ (5 mol\%, $5 \mathrm{mg}$ ), $\mathrm{AgSbF}_{6}(5 \mathrm{~mol} \%, 3.5 \mathrm{mg}$ ), and $\mathrm{CHCl}_{3}(2 \mathrm{~mL})$. The reaction vial was sealed and subsequently heated at $50{ }^{\circ} \mathrm{C}$ for 3-6 h. After completion of the reaction (confirmed by TLC analysis, eluent: $30 \% \mathrm{Et}_{2} \mathrm{O}$ in $\mathrm{CH}_{2} \mathrm{Cl}_{2}$ ), the residue was subjected to silica gel column chromatography $\left(20-40 \% \mathrm{Et}_{2} \mathrm{O}\right.$ in $\left.\mathrm{CH}_{2} \mathrm{Cl}_{2}\right)$ to afford indoloazepinone $\mathbf{6 a}$; yield: $82 \mathrm{mg}$ (86\%); off-white solid; mp 98$100{ }^{\circ} \mathrm{C}$.

${ }^{1} \mathrm{H}$ NMR $\left(300 \mathrm{MHz}\right.$, DMSO- $\left.d_{6}\right): \delta=7.58(\mathrm{br} \mathrm{s}, 1 \mathrm{H}), 7.40(\mathrm{~d}, J=8.28 \mathrm{~Hz}$, $1 \mathrm{H}), 7.34-7.23$ (m, $3 \mathrm{H}), 7.22-7.03(\mathrm{~m}, 4 \mathrm{H}), 6.90-6.76(\mathrm{~m}, 1 \mathrm{H}), 6.33$ $(\mathrm{s}, 1 \mathrm{H}), 6.05(\mathrm{~s}, 1 \mathrm{H}), 4.52-4.05(\mathrm{~m}, 2 \mathrm{H}), 3.82(\mathrm{~s}, 3 \mathrm{H}), 2.39(\mathrm{~s}, 3 \mathrm{H})$, $1.44-1.11(\mathrm{~m}, 8 \mathrm{H}), 0.79(\mathrm{~s}, 9 \mathrm{H})$.

${ }^{13} \mathrm{C}$ NMR $\left(75 \mathrm{MHz}, \mathrm{CDCl}_{3}\right): \delta=168.0,167.6,138.8,138.1,135.7,135.5$, 129.3, 128.7, 128.0, 125.7, 124.1, 123.3, 120.1, 119.4, 116.0, 109.3, $62.1,55.5,51.9,38.7,32.5,31.3,28.6,28.1,23.2$. 


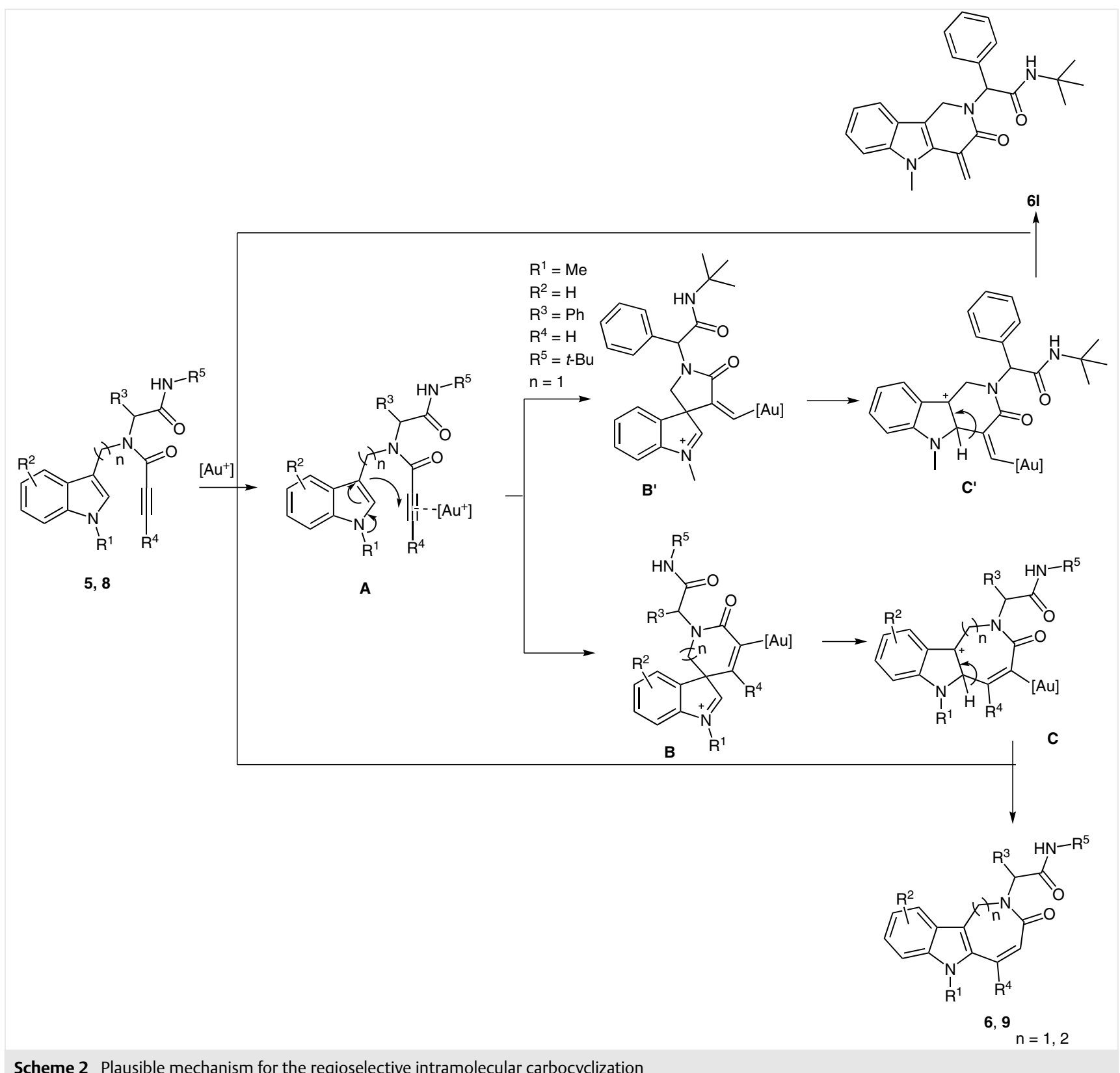

HRMS: $m / z$ calcd for $\mathrm{C}_{30} \mathrm{H}_{37} \mathrm{~N}_{3} \mathrm{O}_{2}$ : 471.2886; found: 471.2863 .

N-tert-Butyl-2-(5,6-dimethyl-3-oxoazepino[4,3-b]indol2(1H,3H,6H)-yl)-2-phenylacetamide (6b)

Compound $\mathbf{6 b}$ was synthesized following the general procedure using 5b (0.2 mmol, $83 \mathrm{mg})$; yield: $66 \mathrm{mg}$ (80\%); off-white solid; mp 143$145^{\circ} \mathrm{C}$.

${ }^{1} \mathrm{H}$ NMR (300 MHz, DMSO- $\left.d_{6}\right): \delta=7.77$ (br s, $\left.1 \mathrm{H}\right), 7.39(\mathrm{~d}, J=8.07 \mathrm{~Hz}$, $1 \mathrm{H}), 7.22-7.05(\mathrm{~m}, 7 \mathrm{H}), 6.89-6.74(\mathrm{~m}, 1 \mathrm{H}), 6.33(\mathrm{~s}, 1 \mathrm{H}), 6.01(\mathrm{~s}, 1 \mathrm{H})$, 4.41-4.10 (m, $2 \mathrm{H}), 3.83$ (s, $3 \mathrm{H}), 2.39(\mathrm{~s}, 3 \mathrm{H}), 1.22(\mathrm{~s}, 9 \mathrm{H})$.
${ }^{13} \mathrm{C}$ NMR $\left(75 \mathrm{MHz}\right.$, DMSO- $\left.d_{6}\right): \delta=166.3,138.0,137.4,136.6,135.1$, $128.6,128.4,127.4,125.6,123.3,122.5,119.0,118.5,115.2,109.6$, $60.5,50.2,38.5,32.1,28.2,22.5$.

HRMS: $m / z$ calcd for $\mathrm{C}_{26} \mathrm{H}_{29} \mathrm{~N}_{3} \mathrm{O}_{2}$ : 415.226; found: 415.2262 .

$\mathrm{N}$-tert-Butyl-2-(5,6-dimethyl-3-oxoazepino[4,3-b]indol$2(1 \mathrm{H}, 3 \mathrm{H}, 6 \mathrm{H})$-yl)pentanamide $(6 \mathrm{c})$

Compound $\mathbf{6 c}$ was synthesized following the general procedure using 5c $(0.2 \mathrm{mmol}, 76 \mathrm{mg})$; yield: $46 \mathrm{mg}(61 \%)$; pale yellow solid; mp 117$119^{\circ} \mathrm{C}$. 
${ }^{1} \mathrm{H}$ NMR $\left(300 \mathrm{MHz}, \mathrm{CDCl}_{3}\right): \delta=7.68(\mathrm{~d}, J=7.89 \mathrm{~Hz}, 1 \mathrm{H}), 7.35-7.26(\mathrm{~m}$, $2 \mathrm{H}), 7.18(\mathrm{t}, J=6.8 \mathrm{~Hz}, 1 \mathrm{H}), 6.42(\mathrm{~s}, 1 \mathrm{H}), 6.01-5.73(\mathrm{br} \mathrm{s}, 1 \mathrm{H}), 4.82(\mathrm{~d}$, $J=7.01 \mathrm{~Hz}, 1 \mathrm{H}), 4.65-4.37(\mathrm{~m}, 1 \mathrm{H}), 4.34-4.12(\mathrm{~m}, 1 \mathrm{H}), 3.84(\mathrm{~s}, 3 \mathrm{H})$, $2.41(\mathrm{~s}, 3 \mathrm{H}), 2.13-1.92(\mathrm{~m}, 1 \mathrm{H}), 1.76-1.59(\mathrm{~m}, 1 \mathrm{H}), 1.29-0.89(\mathrm{~m}, 11$ $\mathrm{H}), 0.80-0.64(\mathrm{~m}, 3 \mathrm{H})$.

${ }^{13} \mathrm{C}$ NMR $\left(75 \mathrm{MHz}, \mathrm{CDCl}_{3}\right): \delta=169.7,167.8,139.0,138.0,135.9,125.6$, 124.0, 123.4, 120.3, 119.4, 116.1, 109.5, 57.3, 51.0, 37.3, 32.4, 30.2, 28.3, 23.2, 19.2, 13.6 .

HRMS: $m / z$ calcd for $\mathrm{C}_{23} \mathrm{H}_{31} \mathrm{~N}_{3} \mathrm{O}_{2}$ : 381.2416; found: 381.2402 .

\section{N-tert-Butyl-2-(5,6-dimethyl-3-oxoazepino[4,3-b]indol- 2(1H,3H,6H)-yl)-3-methylbutanamide (6d)}

Compound $\mathbf{6 d}$ was synthesized following the general procedure using 5d $(0.2 \mathrm{mmol}, 76 \mathrm{mg})$; yield: $60 \mathrm{mg}$ (79\%); white solid; mp 182$184^{\circ} \mathrm{C}$.

${ }^{1} \mathrm{H}$ NMR $\left(300 \mathrm{MHz}\right.$, DMSO- $\left.d_{6}\right): \delta=7.69(\mathrm{~d}, J=7.73 \mathrm{~Hz}, 1 \mathrm{H}), 7.56(\mathrm{br} \mathrm{s}$, $1 \mathrm{H}), 7.48(\mathrm{~d}, J=8.22 \mathrm{~Hz}, 1 \mathrm{H}), 7.20(\mathrm{t}, J=7.49 \mathrm{~Hz}, 1 \mathrm{H}), 7.09(\mathrm{t}, J=7.49$ $\mathrm{Hz}, 1 \mathrm{H}), 6.32(\mathrm{~s}, 1 \mathrm{H}), 4.46$ (d, J = $10.87 \mathrm{~Hz}, 1 \mathrm{H}), 3.86(\mathrm{~s}, 3 \mathrm{H}), 2.39$ (s, 3 H), 2.31-2.13 (m, $1 \mathrm{H}), 1.12(\mathrm{~s}, 9 \mathrm{H}), 0.85(\mathrm{~d}, J=6.35 \mathrm{~Hz}, 3 \mathrm{H}), 0.62-$ $0.10(\mathrm{~m}, 3 \mathrm{H})$.

${ }^{13} \mathrm{C}$ NMR $\left(75 \mathrm{MHz}, \mathrm{CDCl}_{3}\right): \delta=168.9,167.9,138.9,137.9,135.7,125.5$, $124.5,123.2,120.2,119.8,116.2,109.2,63.9,51.1,37.3,32.4,28.3$, 26.7, 23.1, 19.4, 18.6 .

HRMS: $m / z$ calcd for $\mathrm{C}_{23} \mathrm{H}_{31} \mathrm{~N}_{3} \mathrm{O}_{2}$ : 381.2416; found: 381.2415 .

\section{$\mathrm{N}$-Cyclohexyl-2-(5,6-dimethyl-3-oxoazepino[4,3-b]indol- 2(1H,3H,6H)-yl)-2-(4-methoxyphenyl)acetamide (6e)}

Compound $\mathbf{6 e}$ was synthesized following the general procedure using 5e $(0.2 \mathrm{mmol}, 94 \mathrm{mg})$; yield: $70 \mathrm{mg}$ (74\%); off-white solid; mp 120$122^{\circ} \mathrm{C}$.

${ }^{1} \mathrm{H}$ NMR $\left(300 \mathrm{MHz}\right.$, DMSO- $\left.d_{6}\right): \delta=7.99(\mathrm{br} \mathrm{s}, 1 \mathrm{H}), 7.40(\mathrm{~d}, J=7.92 \mathrm{~Hz}$, $1 \mathrm{H}), 7.19-7.00(\mathrm{~m}, 3 \mathrm{H}), 6.95-9.76(\mathrm{~m}, 4 \mathrm{H}), 6.31(\mathrm{~s}, 1 \mathrm{H}), 5.95(\mathrm{~s}, 1 \mathrm{H})$, 4.37-4.12 (m, $1 \mathrm{H}), 3.82(\mathrm{~s}, 3 \mathrm{H}), 3.78-3.66(\mathrm{~m}, 4 \mathrm{H}), 3.62-3.45(\mathrm{~m}, 1$ $\mathrm{H}), 2.38(\mathrm{~s}, 3 \mathrm{H}), 1.76-1.48(\mathrm{~m}, 6 \mathrm{H}), 1.20-0.97(\mathrm{~m}, 4 \mathrm{H})$.

${ }^{13} \mathrm{C} \mathrm{NMR}\left(75 \mathrm{MHz}, \mathrm{CDCl}_{3}\right): \delta=168.5,167.6,159.4,138.7,138.1,135.6$, $130.5,127.3,125.8,124.0,123.3,119.4,116.1,114.3,114.2,109.2$, $55.3,48.2,38.5,32.8,32.4,32.3,25.4,24.7(2), 24.5,23.1$.

HRMS: $m / z$ calcd for $\mathrm{C}_{29} \mathrm{H}_{33} \mathrm{~N}_{3} \mathrm{O}_{3}$ : 471.2522; found: 471.2503 .

\section{$\mathrm{N}$-Butyl-2-cyclohexyl-2-(5,6-dimethyl-3-oxoazepino[4,3-b]indol- $\mathbf{2}(\mathbf{1 H}, \mathbf{3 H}, \mathbf{6 H})$-yl)acetamide (6f)}

Compound $\mathbf{6 f}$ was synthesized following the general procedure using 5f $(0.2 \mathrm{mmol}, 84 \mathrm{mg})$; yield: $62 \mathrm{mg}$ (74\%); pale yellow solid; mp 76$78{ }^{\circ} \mathrm{C}$.

${ }^{1} \mathrm{H}$ NMR $\left(300 \mathrm{MHz}\right.$, DMSO- $\left.d_{6}\right): \delta=8.01$ (br s, $\left.1 \mathrm{H}\right), 7.69(\mathrm{~d}, J=7.14 \mathrm{~Hz}$, $1 \mathrm{H}), 7.49$ (d, J = 8.10 Hz, $1 \mathrm{H}), 7.21(\mathrm{t}, J=7.49 \mathrm{~Hz}, 1 \mathrm{H}), 7.11$ (t, $J=7.49$ $\mathrm{Hz}, 1 \mathrm{H}), 6.31$ (s, $1 \mathrm{H}), 4.53$ (d, J=11.10 Hz, $1 \mathrm{H}), 3.85$ (s, $3 \mathrm{H}), 3.23-$ $2.64(\mathrm{~m}, 2 \mathrm{H}), 2.38(\mathrm{~s}, 3 \mathrm{H}), 2.07-1.85(\mathrm{~m}, 1 \mathrm{H}), 1.69-1.36(\mathrm{~m}, 4 \mathrm{H})$, $1.28-0.63(\mathrm{~m}, 15 \mathrm{H})$.

${ }^{13} \mathrm{C}$ NMR $\left(75 \mathrm{MHz}\right.$, DMSO- $\left.d_{6}\right): \delta=166.2,138.3,137.6,134.4,126.1$, 123.8, 122.6, 119.5, 115.4, 109.9, 60.4, 37.9, 36.3, 32.1, 30.7, 29.1, $28.8,25.7,25.1,24.7,22.4,19.3,13.5$.

HRMS: $m / z$ calcd for $\mathrm{C}_{26} \mathrm{H}_{35} \mathrm{~N}_{3} \mathrm{O}_{2}$ : 421.2729 ; found: 421.2717 .

\section{$\mathrm{N}$-Cyclohexyl-2-(5-ethyl-6-methyl-3-oxoazepino[4,3-b]indol-} 2(1H,3H,6H)-yl)-2-(4-methoxyphenyl)acetamide (6g)

Compound $\mathbf{6 g}$ was synthesized following the general procedure using $\mathbf{5 g}$ (0.2 mmol, $97 \mathrm{mg})$; yield: $75 \mathrm{mg}$ (77\%); light yellow solid; mp 118$119^{\circ} \mathrm{C}$.

${ }^{1} \mathrm{H} \mathrm{NMR}\left(300 \mathrm{MHz}, \mathrm{CDCl}_{3}\right): \delta=7.98($ br s, $1 \mathrm{H}), 7.40(\mathrm{~d}, J=8.28 \mathrm{~Hz}, 1$ H), 7.24-6.89 (m, $4 \mathrm{H}), 6.88-6.63(\mathrm{~m}, 3 \mathrm{H}), 6.33(\mathrm{~s}, 1 \mathrm{H}), 5.96(\mathrm{~s}, 1 \mathrm{H})$, 4.47-4.03 (m, $1 \mathrm{H}), 3.80(\mathrm{~s}, 3 \mathrm{H}), 3.75-3.44(\mathrm{~m}, 5 \mathrm{H}), 2.88-2.61(\mathrm{~m}, 2$ H), 1.83-1.44 (m, $5 \mathrm{H}), 1.33-1.12$ ( $\mathrm{m}, 3 \mathrm{H}), 1.11-0.94(\mathrm{~m}, 5 \mathrm{H})$.

${ }^{13} \mathrm{C}$ NMR $\left(75 \mathrm{MHz}, \mathrm{CDCl}_{3}\right): \delta=167.8,159.4,141.8,138.8,137.4,130.6$, $127.2,124.3,124.1,123.1,119.3,116.6,114.2,109.2,61.6,55.3,48.2$, $38.5,32.3,30.9,29.2,25.4,24.5,22.3,14.0,13.3$.

HRMS: $m / z$ calcd for $\mathrm{C}_{30} \mathrm{H}_{35} \mathrm{~N}_{3} \mathrm{O}_{3}$ : 485.2678; found: 485.2649 .

$N$-tert-Butyl-2-(5-isopropyl-6-methyl-3-oxoazepino[4,3-b]indol2(1H,3H,6H)-yl)-2-phenylacetamide (6h)

Compound $\mathbf{6 h}$ was synthesized following the general procedure using 5h (0.2 mmol, $89 \mathrm{mg}$ ); yield: $53 \mathrm{mg}$ (60\%); white solid; mp 138$140{ }^{\circ} \mathrm{C}$.

${ }^{1} \mathrm{H}$ NMR (400 MHz, DMSO- $\left.d_{6}\right): \delta=7.96(\mathrm{~d}, J=6.1 \mathrm{~Hz}, 1 \mathrm{H}), 7.58$ (br s, 1 H), 7.42-7.33 (m, 3 H), 7.28-7.21 (m, 2 H), 7.08-6.98 (m, 2 H), 6.79$6.66(\mathrm{~m}, 1 \mathrm{H}), 6.58-6.50(\mathrm{~m}, 1 \mathrm{H}), 5.83(\mathrm{~s}, 1 \mathrm{H}), 3.64-3.61(\mathrm{~m}, 1 \mathrm{H})$, $3.19(\mathrm{~d}, J=9.2 \mathrm{~Hz}, 1 \mathrm{H}), 2.59(\mathrm{~s}, 3 \mathrm{H}), 1.71(\mathrm{~d}, J=6.8 \mathrm{~Hz}, 6 \mathrm{H}), 1.25(\mathrm{~s}, 9$ $\mathrm{H})$.

${ }^{13} \mathrm{C}$ NMR $\left(75 \mathrm{MHz}\right.$, DMSO- $\left.d_{6}\right): \delta=168.2,166.5,138.2,137.5,136.4$, 135.1, 128.7, 128.3, 127.4, 125.3, 123.6, 122.4, 119.1, 118.3, 115.1, 109.6, 60.8, 50.5, 39.1, 32.1, 28.1, 25.2, 22.6.

HRMS: $m / z$ calcd for $\mathrm{C}_{28} \mathrm{H}_{33} \mathrm{~N}_{3} \mathrm{O}_{2}$ : 443.2573; found: 443.2582 .

\section{N-tert-Butyl-2-(6-methyl-3-oxo-5-phenylazepino[4,3-b]indol-} 2(1H,3H,6H)-yl)-2-phenylacetamide (6i)

Compound $\mathbf{6 i}$ was synthesized following the general procedure using 5i (0.2 mmol, $95 \mathrm{mg})$; yield: $78 \mathrm{mg}$ (82\%); light yellow solid; mp 180$182^{\circ} \mathrm{C}$.

${ }^{1} \mathrm{H}$ NMR $\left(300 \mathrm{MHz}\right.$, DMSO- $\left.d_{6}\right): \delta=7.88(\mathrm{br} \mathrm{s}, 1 \mathrm{H}), 7.53-7.42(\mathrm{~m}, 3 \mathrm{H})$, 7.40-7.06 (m, $10 \mathrm{H}), 6.93-6.70(\mathrm{~m}, 1 \mathrm{H}), 6.54(\mathrm{~s}, 1 \mathrm{H}), 6.10(\mathrm{~s}, 1 \mathrm{H})$, 4.78-4.14 (m, $2 \mathrm{H}), 3.10(\mathrm{~s}, 3 \mathrm{H}), 1.25(\mathrm{~s}, 9 \mathrm{H})$.

${ }^{13} \mathrm{C}$ NMR $\left(75 \mathrm{MHz}, \mathrm{CDCl}_{3}\right): \delta=168.0,139.5,139.3,138.8,136.3,134.4$, $134.2,129.4,129.1,128.1,128.0,125.7,124.0,123.2,119.4,117.2$, 109.3, 109.2, 51.3, 38.8, 34.1, 32.3, 28.3.

HRMS: $m / z$ calcd for $\mathrm{C}_{31} \mathrm{H}_{31} \mathrm{~N}_{3} \mathrm{O}_{2}$ : 477.2416; found: 477.2439 .

\section{N-tert-Butyl-2-(6-methyl-3-oxo-5-p-tolylazepino[4,3-b]indol- 2(1H,3H,6H)-yl)-2-phenylacetamide (6j)}

Compound $\mathbf{6 j}$ was synthesized following the general procedure using 5j (0.2 mmol, $98 \mathrm{mg})$; yield: $91 \mathrm{mg}$ (93\%); white solid; mp 281$283^{\circ} \mathrm{C}$.

${ }^{1} \mathrm{H}$ NMR (300 MHz, DMSO- $d_{6}$ ): $\delta=7.87$ (br s, $1 \mathrm{H}$ ), 7.35-7.06 (m, 11 $\mathrm{H}), 6.93-6.68(\mathrm{~m}, 1 \mathrm{H}), 6.51(\mathrm{~s}, 1 \mathrm{H}), 6.09(\mathrm{~s}, 1 \mathrm{H}), 4.81-3.97(\mathrm{~m}, 2 \mathrm{H})$, $3.11(\mathrm{~s}, 3 \mathrm{H}), 2.36(\mathrm{~s}, 3 \mathrm{H}), 1.26(\mathrm{~s}, 9 \mathrm{H})$.

${ }^{13} \mathrm{C}$ NMR $\left(75 \mathrm{MHz}\right.$, DMSO- $\left.d_{6}\right): \delta=168.5,166.5,138.5,138.1,136.2$, $135.5,129.4,128.8,128.5,127.6,127.5,125.2,122.7,119.1,116.7$, 109.6, 60.6, 50.3, 32.0, 28.2, 20.7.

HRMS: $m / z$ calcd for $\mathrm{C}_{32} \mathrm{H}_{33} \mathrm{~N}_{3} \mathrm{O}_{2}$ : 491.2573; found: 491.2562 . 
N-tert-Butyl-2-(5-methyl-4-methylene-3-oxo-3,4-dihydro-1Hpyrido[4,3-b]indol-2(5H)-yl)-2-phenylacetamide (61)

Compound $\mathbf{6 1}$ was synthesized following the general procedure using 51 (0.2 mmol, $80 \mathrm{mg})$; yield: $25 \mathrm{mg}$ (31\%); off-white solid; mp 110$112^{\circ} \mathrm{C}$.

${ }^{1} \mathrm{H}$ NMR $\left(300 \mathrm{MHz}, \mathrm{CDCl}_{3}\right): \delta=7.51-7.36(\mathrm{~m}, 5 \mathrm{H}), 7.34-7.27(\mathrm{~m}, 3 \mathrm{H})$, 7.05 (t, J = 7.74 Hz, $1 \mathrm{H}), 6.50(\mathrm{~s}, 1 \mathrm{H}), 6.42(\mathrm{~s}, 1 \mathrm{H}), 5.91(\mathrm{~s}, 1 \mathrm{H}), 5.77$ (br s, $1 \mathrm{H}), 5.01(\mathrm{~d}, J=17.31 \mathrm{~Hz}, 1 \mathrm{H}), 4.23(\mathrm{~d}, J=17.52 \mathrm{~Hz}, 1 \mathrm{H}), 3.90(\mathrm{~s}$, $3 \mathrm{H}), 1.38$ (s, $9 \mathrm{H})$.

${ }^{13} \mathrm{C}$ NMR $\left(75 \mathrm{MHz}, \mathrm{CDCl}_{3}\right): \delta=168.5,164.5,139.6,134.8,130.3,129.3$, 129.2, 128.9, 128.5, 123.7, 123.5, 119.7, 118.8, 115.4, 109.3, 108.7, 61.6, 51.8, 42.6, 32.0, 28.6 .

HRMS: $m / z$ calcd for $\mathrm{C}_{25} \mathrm{H}_{27} \mathrm{~N}_{3} \mathrm{O}_{2}$ : 401.2103; found: 401.2080 .

(Z)-N-tert-Butyl-3,3-dimethyl-2-(4-oxo-6-phenyl-1H-azocino[5,4b]indol-3(2H,4H,7H)-yl)butanamide (9a); Typical Procedure

In a $10 \mathrm{~mL}$ screw cap vial charged with Ugi adduct $8 \mathbf{8}(0.2 \mathrm{mmol}, 91$ mg) was added $\mathrm{AuPPh}_{3} \mathrm{Cl}$ (5 mol\%, $5 \mathrm{mg}$ ), $\mathrm{AgSbF}_{6}$ ( $5 \mathrm{~mol} \%, 3.5 \mathrm{mg}$ ), and $\mathrm{CHCl}_{3}(2 \mathrm{~mL})$. The vial was sealed and subsequently heated at $50{ }^{\circ} \mathrm{C}$ for 3-5 h. After completion of the reaction (confirmed by TLC analysis, eluent: $30 \% \mathrm{Et}_{2} \mathrm{O}$ in $\mathrm{CH}_{2} \mathrm{Cl}_{2}$ ), the residue was subjected to silica gel column chromatography (using $10-30 \% \mathrm{Et}_{2} \mathrm{O}-\mathrm{CH}_{2} \mathrm{Cl}_{2}$ ) to afford indoloazepinone 9a; yield: $73 \mathrm{mg}(80 \%)$; white solid; $\mathrm{mp} 220-222^{\circ} \mathrm{C}$.

${ }^{1} \mathrm{H}$ NMR (300 MHz, $\mathrm{CDCl}_{3}$ ): $\delta=10.58$ (s, $1 \mathrm{H}$ ), 7.85 (br s, $1 \mathrm{H}$ ), 7.49$7.25(\mathrm{~m}, 7 \mathrm{H}), 7.20$ (d, $J=8.10 \mathrm{~Hz}, 1 \mathrm{H}), 7.07$ (d, $J=7.53 \mathrm{~Hz}, 1 \mathrm{H}), 6.96$ $(\mathrm{t}, J=7.53 \mathrm{~Hz}, 1 \mathrm{H}), 6.43(\mathrm{~s}, 1 \mathrm{H}), 4.97-4.78(\mathrm{~m}, 1 \mathrm{H}), 4.41-4.17(\mathrm{~m}, 2$ H), 2.99-2.79 (m, $1 \mathrm{H}), 1.23(\mathrm{~s}, 9 \mathrm{H}), 0.99(\mathrm{~s}, 9 \mathrm{H})$.

${ }^{13} \mathrm{C}$ NMR $\left(75 \mathrm{MHz}, \mathrm{CDCl}_{3}\right): \delta=172.2,170.7,168.5,139.3,135.8,129.7$, 129.0, 128.8, 128.6, 128.4, 123.2, 122.7, 119.5, 119.0, 112.1, 110.6, 51.3, 35.4, 31.8, 28.3, 26.4, 22.6, 14.1.

HRMS: $m / z$ calcd for $\mathrm{C}_{29} \mathrm{H}_{35} \mathrm{~N}_{3} \mathrm{O}_{2}$ : 457.2729; found: 457.2706.

(Z)-N-Butyl-2-(2,6-dichlorophenyl)-2-(6-methyl-4-oxo-1H-azocino[5,4-b] indol-3 $(2 H, 4 H, 7 H)$-yl)acetamide $(9 b)$

Compound $\mathbf{9 b}$ was synthesized following the general procedure using the readily synthesized Ugi adduct $\mathbf{8 b}(0.2 \mathrm{mmol}, 97 \mathrm{mg})$ and the data for this compound were found in accordance with the literature report; ${ }^{10}$ yield: $78 \mathrm{mg}(80 \%)$; white solid; $\mathrm{mp} 250-253^{\circ} \mathrm{C}$.

${ }^{1} \mathrm{H}$ NMR (300 MHz, DMSO- $\left.d_{6}\right): \delta=10.8(\mathrm{~s}, 1 \mathrm{H}), 7.95-7.66(\mathrm{~m}, 1 \mathrm{H})$, 7.50-7.31 (m, $3 \mathrm{H}), 7.25(\mathrm{~d}, J=8.22 \mathrm{~Hz}, 1 \mathrm{H}), 7.07(\mathrm{t}, J=7.22 \mathrm{~Hz}, 1 \mathrm{H})$, $6.88(\mathrm{t}, J=7.45 \mathrm{~Hz}, 1 \mathrm{H}), 6.80-6.69(\mathrm{~m}, 1 \mathrm{H}), 6.37(\mathrm{~s}, 1 \mathrm{H}), 5.97(\mathrm{~s}, 1 \mathrm{H})$, 4.35-4.07 (m, $1 \mathrm{H}), 3.96-3.82(\mathrm{~m}, 1 \mathrm{H}), 3.10-2.90(\mathrm{~m}, 2 \mathrm{H}), 2.76-2.54$ (m, $1 \mathrm{H}), 2.28$ (s, $3 \mathrm{H}), 2.04-1.88(\mathrm{~m}, 1 \mathrm{H}), 1.40-1.10$ (m, $4 \mathrm{H}), 0.88-$ $0.78(\mathrm{~m}, 3 \mathrm{H})$.

${ }^{13} \mathrm{C}$ NMR $\left(75 \mathrm{MHz}\right.$, DMSO- $\left.d_{6}\right): \delta=168.7,167.1,135.7,134.2,132.0$, 131.8, 130.6, 129.4, 128.0, 122.0, 121.9, 118.8, 117.8, 110.6, 107.7, 57.3, 44.2, 30.5, 24.8, 23.6, 19.5, 13.6 .

HRMS: $m / z$ calcd for $\mathrm{C}_{26} \mathrm{H}_{27} \mathrm{Cl}_{2} \mathrm{~N}_{3} \mathrm{O}_{2}$ : 483.1480; found: 483.1468 .

(Z)-N-tert-Butyl-2-cyclohexyl-2-(10-methoxy-6-methyl-4-oxo-1Hazocino[5,4-b]indol-3(2H,4H,7H)-yl)acetamide (9c)

Compound $9 \mathbf{c}$ was synthesized following the general procedure using the readily synthesized Ugi adduct $8 \mathbf{c}(0.2 \mathrm{mmol}, 90 \mathrm{mg})$ and the data of this compound were found in accordance with the literature report; ${ }^{10}$ yield: $59 \mathrm{mg}$ (65\%); white solid; $\mathrm{mp} 218-220^{\circ} \mathrm{C}$.
${ }^{1} \mathrm{H}$ NMR $\left(300 \mathrm{MHz}\right.$, DMSO- $\left.d_{6}\right): \delta=10.64(\mathrm{~s}, 1 \mathrm{H}), 7.53$ (br s, $1 \mathrm{H}$ ), 7.16 (d, $J=8.69 \mathrm{~Hz}, 1 \mathrm{H}), 6.79$ (s, $1 \mathrm{H}), 6.72(\mathrm{dd}, J=1.70,8.75 \mathrm{~Hz}, 1 \mathrm{H}), 5.88$ (s, $1 \mathrm{H}), 4.62-4.49(\mathrm{~m}, 1 \mathrm{H}), 4.20-4.07(\mathrm{~m}, 1 \mathrm{H}), 3.99-3.85(\mathrm{~m}, 1 \mathrm{H})$, $3.71(\mathrm{~s}, 3 \mathrm{H}), 3.14-2.80(\mathrm{~m}, 2 \mathrm{H}), 2.20(\mathrm{~s}, 3 \mathrm{H}), 2.02-1.88(\mathrm{~m}, 1 \mathrm{H})$, 1.72-1.50 (m, $3 \mathrm{H}), 1.49-1.17$ (m, $3 \mathrm{H}), 1.12$ (s, $9 \mathrm{H}), 1.08-0.79$ (m, 3 $\mathrm{H})$.

${ }^{13} \mathrm{C}$ NMR $\left(75 \mathrm{MHz}, \mathrm{CDCl}_{3}\right): \delta=170.8,169.4,154.0,134.5,132.1,131.0$, 129.2, 121.4, 113.7, 111.4, 109.8, 100.2, 55.8, 51.0, 35.6, 30.2, 29.1, 28.3, 26.3, 25.7 (2), 23.9.

HRMS: $m / z$ calcd for $\mathrm{C}_{27} \mathrm{H}_{37} \mathrm{~N}_{3} \mathrm{O}_{3}$ : 451.2835 ; found: 451.2847 .

(Z)-N-Cyclohexyl-2-[6-(4-methoxyphenyl)-4-oxo-1H-azocino[5,4b]indol-3(2H,4H,7H)-yl]-2-phenylacetamide (9d)

Compound 9d was synthesized following the general procedure using 8d (0.2 mmol, $107 \mathrm{mg})$; yield: $97 \mathrm{mg}(91 \%)$; white solid; mp 271$273^{\circ} \mathrm{C}$.

${ }^{1} \mathrm{H}$ NMR (300 MHz, DMSO- $\left.d_{6}\right): \delta=10.59(\mathrm{~s}, 1 \mathrm{H}), 7.74(\mathrm{~d}, J=7.6 \mathrm{~Hz}, 1$ H), 7.46-7.04 (m, $9 \mathrm{H}), 7.01-6.91(\mathrm{~m}, 4 \mathrm{H}), 6.35(\mathrm{~s}, 1 \mathrm{H}), 5.87(\mathrm{~s}, 1 \mathrm{H})$, 4.42-4.07 (m, $1 \mathrm{H}), 3.93-3.64(\mathrm{~m}, 4 \mathrm{H}), 3.58-3.45(\mathrm{~m}, 1 \mathrm{H}), 2.49-2.31$ (m, $1 \mathrm{H}), 2.12-1.81(\mathrm{~m}, 1 \mathrm{H}), 1.69-1.43(\mathrm{~m}, 5 \mathrm{H}), 1.28-1.11(\mathrm{~m}, 2 \mathrm{H})$, $1.08-0.70(\mathrm{~m}, 3 \mathrm{H})$.

${ }^{13} \mathrm{C}$ NMR $\left(75 \mathrm{MHz}\right.$, DMSO- $\left.d_{6}\right): \delta=169.2,159.7,136.8,136.0,131.6$, $130.4,129.3,129.1,128.4,127.8,122.0,121.1,118.3,118.1,113.8$, 111.0, 110.5, 55.2, 54.8, 47.6, 32.0, 25.0, 24.5, 24.4.

HRMS: $m / z$ calcd for $\mathrm{C}_{34} \mathrm{H}_{35} \mathrm{~N}_{3} \mathrm{O}_{3}$ : 533.2678; found: 533.2677 .

(Z)-N-tert-Butyl-2-(10-methoxy-4-oxo-6-phenyl-1H-azocino[5,4b]indol-3(2H,4H,7H)-yl)pentanamide (9e)

Compound 9e was synthesized following the general procedure using 8e (0.2 mmol, $95 \mathrm{mg})$; yield: $80 \mathrm{mg}$ (84\%); off-white solid; mp 258$260{ }^{\circ} \mathrm{C}$.

${ }^{1} \mathrm{H}$ NMR (300 MHz, THF- $d_{8}$ ): $\delta=9.46$ (s, $1 \mathrm{H}$ ), 7.47 (br s, $1 \mathrm{H}$ ), $7.33-$ $7.12(\mathrm{~m}, 5 \mathrm{H}), 6.92(\mathrm{~d}, J=8.64 \mathrm{~Hz}, 1 \mathrm{H}), 6.84(\mathrm{~s}, 1 \mathrm{H}), 6.61(\mathrm{~d}, J=8.28$ $\mathrm{Hz}, 1 \mathrm{H}), 6.51$ (br s, $1 \mathrm{H}), 6.18(\mathrm{~s}, 1 \mathrm{H}), 4.59-4.41(\mathrm{~m}, 1 \mathrm{H}), 4.07-3.91$ (m, $1 \mathrm{H}), 3.67$ (s, $3 \mathrm{H}), 3.28-2.90(\mathrm{~m}, 2 \mathrm{H}), 1.98-1.76(\mathrm{~m}, 1 \mathrm{H}), 1.59-$ $1.46(\mathrm{~m}, 1 \mathrm{H}), 1.25-1.02(\mathrm{~m}, 2 \mathrm{H}), 0.94(\mathrm{~s}, 9 \mathrm{H}), 0.77$ (t, J = $7.05 \mathrm{~Hz}, 3$ $\mathrm{H})$.

${ }^{13} \mathrm{C}$ NMR $\left(75 \mathrm{MHz}, \mathrm{THF}-d_{8}\right): \delta=167.4(2), 152.2,138.7,136.1,129.9$, 128.8, 127.1, 126.4, 126.2 (2), 121.1, 111.3, 109.4, 97.6, 52.9, 48.2, 29.1, 25.8, 24.0, 17.6, 11.6.

HRMS: $m / z$ calcd for $\mathrm{C}_{29} \mathrm{H}_{35} \mathrm{~N}_{3} \mathrm{O}_{3}$ : 473.2673; found: 473.2663 .

\section{Acknowledgment}

The authors wish to thank the F.W.O [Fund for Scientific Research Flanders (Belgium)] and the Research Fund of the University of Leuven (KU Leuven) for financial support. D.D.V. is thankful to EMECW, Lot 13 (Erasmus Mundus External Cooperation Window, Lot 13) for providing a doctoral scholarship and A.K. is thankful to EMA2 experts (Erasmus Mundus Action 2, Lot 11 Asia: Experts) for providing a doctoral exchange scholarship. The authors thank Ir. B. Demarsin for HRMS measurements.

\section{Supporting Information}

Supporting information for this article is available online at http://dx.doi.org/10.1055/s-0034-1379894. 


\section{References}

(1) Present address: Chemistry Building-4.20b, School of Chemistry, The University of Manchester, Manchester M13 9PL, UK.

(2) Newman, D. J.; Cragg, G. M.; Snader, K. M. J. Nat. Prod. 2003, 66, 1022.

(3) (a) Magolan, J.; Kerr, M. A. Org. Lett. 2006, 8, 4561. (b) Kam, T.S.; Sim, K.-M.; Lim, T.-M. Tetrahedron Lett. 2000, 41, 2733.

(4) (a) Sausville, E. A.; Zaharevitz, D.; Gussio, R.; Meijer, L.; LouranLeost, M.; Kunick, C.; Schultz, R.; Lahusen, T.; Headlee, D.; Stinson, S.; Arbuck, S. G.; Senderowicz, A. Pharmacol. Ther. 1999, 82, 285. (b) Zaharevitz, D. Z.; Gussio, R.; Leost, M.; Senderowicz, A.; Lahusen, T.; Kunick, C.; Meijer, L.; Sausville, E. A. Cancer Res. 1999, 59, 2566.

(5) (a) Irlinger, B.; Bartsch, A.; Kramer, H.-J.; Mayser, P.; Steglich, W. Helv. Chim. Acta 2005, 88, 1472. (b) Mayser, P.; Wenzel, M.; Kramer, H.-J.; Kindler, B. L. J.; Spiteller, P.; Haase, G. Med. Mycol. 2007, 45, 519. (c) Zuther, K.; Mayser, P.; Hettwer, U.; Wu, W.; Spiteller, P.; Kindler, B. L. J.; Karlovsky, P.; Basse, C. W.; Schirawski, J. Mol. Microbiol. 2008, 68, 152.

(6) Sharma, V.; Lansdell, T. A.; Jin, G.; Tepe, J. J. J. Med. Chem. 2004, $47,3700$.

(7) (a) Frank, K. E.; Fu, J-m.; Acker, B. A.; Ennis, M. D.; Fisher, J. F.; Jacobsen, E. J.; McWhorter, W. W.; Morris, J. K.; Rogier, D. J. Jr.; (Upjohn Co.) Patent WO 02/24700, 2002; Chem. Abstr. 2002, 136, 279440p. (b) Frank, K. E.; Fu, J-m.; Acker, B. A.; Ennis, M. D.; Fisher, J. F.; Jacobsen, E. J.; McWhorter, W. W.; Morris, J. K.; Rogier, D. J. Jr.; (Upjohn Co.) Patent WO 02/24701, 2002; Chem. Abstr. 2002, 136, 279356r. (c) Kraxner, J.; Hubner, H.; Gmeiner, P. Arch. Pharm. Pharm. Med. Chem. 2000, 333, 287.

(8) (a) Donets, P. A.; Van der Eycken, E. V. Synthesis 2011, 2147. (b) Gruit, M.; Pews-Davtyan, A.; Beller, M. Org. Biomol. Chem. 2011, 9, 1148. (c) Stuart, D. R.; Alsabeh, P.; Kuhn, M.; Fagnou, K. J. Am. Chem. Soc. 2010, 132, 18326. (d) Reyes-Gutiérrez, P. E.; Torres-Ochoa, R. O.; Martínez, R.; Miranda, L. D. Org. Biomol. Chem. 2009, 7, 1388. (e) Power, D. P.; Lozach, O.; Meijer, L.; Grayson, D. H.; Connon, S. J. Bioorg. Med. Chem. Lett. 2010, 20, 4940. (f) White, A. W.; Carpenter, N.; Lottin, J. R. P.; McClelland, R. A.; Nicholson, R. I. Eur. J. Med. Chem. 2012, 56, 246.

(9) For the synthesis of indoloazepines using isonitrile based multicomponent reaction, see: (a) Zhang, L.; Zheng, M.; Zhao, F.; Zhai, Y.; Liu, H. ACS Comb. Sci. 2014, 16, 184. (b) Subba Reddy, B. S.; Ganesh, A. V.; Vani, M.; Murthi, T. R.; Kalivendi, S. V.; Yadav, J. S. Bioorg. Med. Chem. Lett. 2014, 24, 4501. (c) Zhang, L.; Zhao, F.; Zheng, M.; Zhai, Y.; Liu, H. Chem. Commun. 2013, 49, 2894. (d) Beaumont, S.; Retailleau, P.; Dauban, P.; Dodd, R. H. Eur. J. Org. Chem. 2008, 5162. (e) Gordillo-Cruz, R. E.; Rentería-Gómez, A.; Islas-Jácome, A.; Cortes-García, C. J.; Díaz-Cervantes, E.; Robles, J.; Gámez-Montaño, R. Org. Biomol. Chem. 2013, 11, 6470. (f) Jida, M.; Betti, C.; Urbanczyk-Lipkowska, Z.; Tourwé, D.; Ballet, S. Org. Lett. 2013, 15, 5866.
(10) (a) Modha, S. G.; Kumar, A.; Vachhani, D. D.; Jacobs, J.; Sharma, S. K.; Parmar, V. S.; Van Meervelt, L.; Van der Eycken, E. V. Angew. Chem. Int. Ed. 2012, 51, 9572. (b) Modha, S. G.; Kumar, A.; Vachhani, D. D.; Sharma, S. K.; Parmar, V. S.; Van der Eycken, E. V. Chem. Commun. 2012, 48, 10916. (c) Dyker, G. Angew. Chem. Int. Ed. 2000, 39, 4237. (d) Hashmi, A. S. K.; Hutchings, G. Angew. Chem. Int. Ed. 2006, 45, 7896. (e) Hashmi, A. S. K. Chem. Rev. 2007, 107, 3780. (f) Fürstner, A.; Davies, P. W. Angew. Chem. Int. Ed. 2007, 46, 3410. (g) Gorin, D. J.; Sherry, B. D.; Toste, F. D. Chem. Rev. 2008, 108, 3351. (h) Jiménez-Númez, E.; Echavarren, A. M. Chem. Rev. 2008, 108, 3326. (i) Li, Z. G.; Brouwer, C.; He, C. Chem. Rev. 2008, 108, 3239. (j) Arcadi, A. Chem. Rev. 2008, 108, 3266. (k) Hashmi, A. S. K.; Rudolph, M. Chem. Soc. Rev. 2008, 37, 1766. (l) Rudolph, M.; Hashmi, A. S. K. Chem. Soc. Rev. 2012, 41, 2448. (m) Echavarren, A. M. Nat. Chem. 2009, 1, 431. (n) Jiménez-Númez, E.; Echavarren, A. M. Chem. Commun. 2007, 333. (o) Rudolph, M.; Hashmi, A. S. K. Chem. Commun 2011, 47, 6536. (p) Vachhani, D. D.; Modha, S. G.; Sharma, A.; Van der Eycken, E. V. Tetrahedron 2013, 69, 359.

(11) Modha, S. G.; Vachhani, D. D.; Jacobs, J.; Van Meervelt, L.; Van der Eycken, E. V. Chem. Commun. 2012, 48, 6550.

(12) (a) Vachhani, D. D.; Mehta, V. P.; Modha, S. G.; Van Hecke, K.; Van Meervelt, L.; Van der Eycken, E. V. Adv. Synth. Catal. 2012, 354, 1593. (b) Vachhani, D. D.; Sharma, A.; Van der Eycken, E. J. Org. Chem. 2012, 77, 8768. (c) Sharma, A.; Vachhani, D.; Van der Eycken, E. Org. Lett. 2012, 14, 1854. (d) Sharma, A.; Vachhani, D.; Van der Eycken, E. Chem. Eur. J. 2013, 19, 1158. (e) Modha, S. G.; Trivedi, J. C.; Mehta, V. P.; Ermolat'ev, D. S.; Van der Eycken, E. V. J. Org. Chem. 2011, 76, 846. (f) Modha, S. G.; Mehta, V. P.; Ermolat'ev, D.; Balzarini, J.; Van Hecke, K.; Van Meervelt, L.; Van der Eycken, E. V. Mol. Divers. 2010, 14, 767.

(13) (a) Vachhani, D. D.; Sharma, A.; Van der Eycken, E. Angew. Chem. Int. Ed. 2013, 52, 2547. (b) Vachhani, D. D.; Kumar, A.; Modha, S. G.; Sharma, S. K.; Parmar, V. S.; Van der Eycken, E. V. Eur. J. Org. Chem. 2013, 7, 1223. (c) Mehta, V. P.; Modha, S. G.; Ruijter, E.; Van Hecke, K.; Van Meervelt, L.; Pannecouque, C.; Balzarini, J.; Orru, R. V. A.; Van der Eycken, E. V. J. Org. Chem. 2011, 76, 2828.

(14) (a) Ugi, I.; Meyr, R.; Fetzer, U.; Steinbrucker, C. Angew. Chem. 1959, 71, 386. (b) Marcaccini, S.; Torroba, T. Nat. Protoc. 2007, 2, 632. (c) Ruijter, E.; Scheffelaar, R.; Orru, R. V. A. Angew. Chem. Int. Ed. 2011, 50, 6234.

(15) (a) Ferrer, C.; Echavarren, A. M. Angew. Chem. Int. Ed. 2006, 45, 1105. (b) Ferrer, C.; Amijs, C. H. M.; Echavarren, A. M. Chem. Eur. J. 2007, 13, 1358. (c) Echavarren, A. M. Nat. Chem. 2009, 1, 431.

(16) For the synthesis of indoloazocines using isonitrile based multicomponent reaction, see: (a) Sinha, M. K.; Khoury, K.; Herdtweck, E.; Dömling, A. Chem. Eur. J. 2013, 19, 8048. (b) Schultz, E. E.; Pujanauski, B. G.; Sarpong, R. Org. Lett. 2012, 14,648 .

(17) For a review on the mechanistic aspects of gold-catalyzed reactions, see: Hashmi, A. S. K. Angew. Chem. Int. Ed. 2010, 49, 5232. 